\title{
Graphic Composition Art of Packaging Visual Communication Design Based on VR
}

\author{
Yanna Wang $\mathbb{D}$ \\ School of Art and Media, Xi'an Technological University, Xian, Shaanxi 710032, China \\ Correspondence should be addressed to Yanna Wang; wangyanna@xatu.edu.cn
}

Received 7 January 2022; Revised 23 January 2022; Accepted 26 January 2022; Published 11 February 2022

Academic Editor: Xin Ning

Copyright (c) 2022 Yanna Wang. This is an open access article distributed under the Creative Commons Attribution License, which permits unrestricted use, distribution, and reproduction in any medium, provided the original work is properly cited.

\begin{abstract}
Visual communication is a popular art form that aids in the transmission of information and reflects people's aesthetic preferences. In visual communication, the graphic element is an important combination element that plays a key role. Virtual reality (VR) technology will become more widely used as computer technology advances and network applications become more popular. As a result, research into VR technology generation and application in packaging design will aid in the popularization and application of VR technology, as well as the advancement of product packaging design. The application of graphic composition art in packaging visual communication design is investigated in this paper. This paper introduces the basic concepts and characteristics of virtual reality (VR), as well as the development and application fields of VR technology, based on the current state of virtual panorama development at home and abroad. It also provides advice and recommendations on graphic creativity in VR technology packaging design, as well as the symbol of graphic creativity and the value of graphic composition art in packaging visual communication design.
\end{abstract}

\section{Introduction}

Digital 3D panorama technology, which is based on sequential pictures, stitches together static images to create a 360 -degree panoramic image that accurately reproduces the real scene [1]. These new features give us a fresh perspective and give visitors a genuine sense of "being there." Since its inception, the design has evolved from its original functions of protection and transportation to that of a brand carrier in the marketing era [2]. The design of the packaging is an important part of visual communication. However, since the gradual commercialization of packaging, it has strayed from the single coverage of visual communication design and injected new life into marketing and branding. Packaging can no longer be defined solely in terms of plane range. Packaging has evolved into a brand symbol [3]. People's lives are constantly influenced by visual communication design. Nowadays, it has penetrated into every aspect of people's daily life, not only reflecting people's lifestyle characteristics but also influencing people's life behavior. With the social progress and the accelerated pace of people's lives, people's demand for information and other aspects has greatly increased, and they no longer just stay in the traditional mode of obtaining information by printing products but instead want various shortcuts to obtain the latest information anytime, anywhere [4]. At present, with the continuous development and progress of science and technology, people's aesthetic concept has also undergone great changes. In the visual communication design, people's aesthetic taste has also changed to some extent. Therefore, visual communication design must keep pace with the times [5]. Among them, the significance of graphic composition art in visual communication is very critical, and it is closely related to visual communication design. It is very important to explore the application of graphic composition art in visual communication design.

Packaging serves to protect goods while also encouraging sales. It is necessary to reproduce commodities in graphic form in order to generate visual demand for consumers [6] so that they can directly understand the contents of commodity packaging. The graphics on the package are intended to help consumers understand the shape and color 
of the goods in the package, to give them an intuitive impression of the goods, and to make them feel the goods' unique humanistic care, in order to motivate them to buy more. The pursuit of visual beauty and other sensory stimuli has become a factor that must be considered in modern packaging design [7]. The function of modern packaging is not only to transmit information but also to bring people the enjoyment of beauty. Packaging design's literary goal is to communicate visual design. Visual composition and visual language, of course, will be part of the design. Visually sensing pictures can be used to make products more appealing. Visually sensing words [8] allow you to sense the connotation and humanity of products. Packaging aspires to resonate with consumers on a spiritual level by shaping the soul and spirit of industrial culture and then allowing them to pay for their spiritual enjoyment and taste. Graphic art and visual communication design for packaging are both external and interdependent. It is designed as a table and composed as Pochtar and Malakhov [9]. Packaging visual communication design is nothing but a dead letter if it lacks the art of graphic composition. In the same way, graphic composition art is useless without packaging visual communication design. This paper studies the graphic composition art of packaging visual communication design based on virtual reality (VR).

With the development of the commodity economy, objective demand puts forward more requirements for packaging, and it is necessary to participate in the competition of market circulation [3]. The new design language is naturally integrated into the commercial design concept, which contributes to the greater success of marketing. The market is gradually developing in the direction of collectivization and supercalculation, which leads to the constant renewal of design concept and form [10]. Exquisite packaging design plays an important role in the process of production and marketing. For modern packaging design, packaging design plays an increasingly important role in product sales [11]. In order to enable consumers to intuitively understand the product features and leave a deep impression on the products, thus generating a strong desire to buy, the means of brand image design integrated with graphics and words on the packaging are becoming more and more abundant. The product packaging design should pay attention to graceful beauty; that is, simplicity is the best. Pay attention to the beauty of the image, that is, rich connotation [12]. Therefore, the quality of graphic composition is closely related to the requirements of packaging visual communication design, or it must be treated as art rather than technology. Particularly rare design is to express rich brand image content in concise packaging graphic design. It is not difficult to find that the art of graphic composition plays a vital role in the visual communication of packaging. Therefore, based on the theoretical research of VR technology, this paper focuses on practical application. Through practical application, the application value of VR technology, the key technologies of digital 3D panorama technology, and the research contents of $3 \mathrm{D}$ panorama packaging design and display are completed. This paper probes into the art of graphic composition in packaging visual communication design.

\section{Related Work}

Packaging, according to the literature [13], has two meanings: one refers to a series of activities involved in the design and manufacture of containers or wrappers, and the other refers to the container or wrapper itself. The literature [14] begins with people's perceptions and perceptions, then combines product packaging design characteristics, and finally takes the practical application of multisensory experience, for example, a more in-depth examination of how people's visual sensory needs for packaging design change over time, the desire for sensory stimulation, and also, research into how to develop and apply the visual space effect in multisensory experience packaging design in a reasonable manner. Engineering and aesthetics should be linked in packaging design, according to literature [15], and both practicality and artistic beauty should be considered. It is a synthesis of several disciplines with the themes of achievement, practicality, and art and culture running through it. Packaging design, according to literature [14], is an important cultural product. Whatever kind of packaging design is the designer's cognition and summary of the product, the content is rich and changeable. From the practical application of the following four aspects, literature [16] analyzes the application of the interactive concept in packaging design: (1) packaging structure interaction, (2) packaging visual image interaction, (3) packaging usage methods interacting with one another, and (4) interaction between the packaging and the user's experience. It also examines the interaction concept's expression form and realization principle in the four applications mentioned above. The article [17] combines comparative analysis and historical analysis in order to gain a better understanding of the evolution of visual communication design. Comparing and contrasting the development of Chinese and foreign visual communication design in order to better recognize the research object, broaden the research perspective, and begin an in-depth investigation of the subject, literature [18] has done a detailed research on packaging design with interactive concepts in the current packaging design field and analyzed the application of interactive concepts in packaging design at this stage. The analysis shows that there are interactive concepts in packaging structure design, packaging visual image design, packaging opening methods, and packaging users'experience of packaging. Literature [19] focuses on the combination of consumer perception, perceptual psychological process, and the visual space of packaging design with the concept of "multisensory" as the main research object. Its purpose is to study the selection and characteristics of elements that affect the packaging of multisensory experience packaging, such as the shape, structure, color, and packaging material design. At the same time, it also includes the influence of visual elements on other senses, as well as the design performance and application of visual space and senses in virtual packaging design in the new media era. Literature [20] explored the relationship between graphic composition art and visual communication design and how to apply graphic composition art in visual communication design. He also pointed 
out that in the process of specific visual communication design, designers should focus on the artistic elements of graphics and integrate them into the works better, making the works more aura and vitality. Literature [9] aimed at the problem of poor element integration performance in traditional packaging visual graphic element integration systems and designed a packaging visual graphic element integration system based on VR. The application environment of virtual packaging - the attributes of virtual space and the new characteristics of interpersonal communication in virtual space-was investigated in literature [21]. Concentrate on the form composition and sensory elements of virtual packaging, which is a new concept, and classify the virtual packaging manifestations that contain these elements. According to literature [22], no design activity is solely for the purpose of design. The ultimate goal is to convey visual information in a clear and effective manner, as well as to obtain feedback through effective appeals. This set of behaviors is organically consistent, interactive, and inextricably linked to the media. According to literature [23], the form of expression for information transmission and ideology influences graphic creativity in packaging design. The form of expression is a type of modeling that is organized into a grammatical structure by a plethora of forms. The imagination can be concretized and visualized with the help of appropriate and effective media, forms, and methods in order to achieve content unity. As a result, unique visual language and formal structure in packaging graphic design must be studied. The literature [24] is based on the fundamental theories of omnimedia and visual communication design, using semiotics, design, psychology, marketing, communication, and other theories as the foundation, and employing the methods of literature consultation and practice summaries to comprehend the omnimedia. The article's arguments are based on the various development forms of visual communication design in the era. Utilize the survey data to identify typical cases, analyze and summarize the thesis's arguments, and gradually organize them into articles. Literature [25] starts from the principle of packaging visual communication design and divides visual communication design into two-dimensional graphic design. Starting from the perspective of brand effect, through the promotion effect of packaging design on the brand, it analyzes the packaging cases of well-known sportswear brands at home and abroad. Combining computer graphic design software to use it in practice, it has received good market feedback. Through the discussion of graphic composition art in packaging visual communication design, the article reveals the important position of graphic design in commodity packaging. The geometric model of camera imaging and the resulting transformation model between images are understood theoretically, and some basic knowledge that should be mastered in image mosaic is introduced. In VR technology, the commonly used panorama generation technology will be studied. The core technologies of image mosaic mainly include image registration technology and image combination technology. This paper discusses the rules of beauty composed of graphic creativity and graphic composition: graphic association and imagination, culture and graphic symbol, morphological isomorphism, morphological reconstruction, decorative character morphology, and so on. The research on the generation technology of VR technology and the application of VR in packaging design in this paper will contribute to the popularization and application of VR technology and the further development of product packaging design.

\section{Methodology}

3.1. VR Technology. VR, also known as spiritual environment technology, is a lifelike 3D VR environment built by people using computer technology, which is a new technology that allows users to simulate vision, hearing, touch, and other senses through computer input devices, which is one of the big technologies. Now, we have entered the era of all media [26]. In the all-media era, new and old media are developed and integrated. The media is highly developed, with rich media, diverse sources of information, and rich content. It serves mankind through all-media, all-inclusive, all-sensory, and all-time communication methods. Human aesthetic needs, information needs, commodity needs, technology drive, audience segmentation, and policy support have enabled the rapid development of all media and its global popularity. VR has three main characteristics: interactivity, immersion, and imagination. The composition of VR is shown in Figure 1.

In essence, VR technology is an advanced and digital interface between humans and computers. It can provide users with a $3 \mathrm{D}$ virtual environment that combines comprehensive perception of vision, hearing, and touch. Users are in this environment, interact with this simulated environment, get all kinds of information contained in the virtual environment, synthesize their own perception and knowledge, and finish their work in this environment most efficiently and conveniently. VR technology is a combination of software and hardware, which includes computer graphics, image processing, and pattern recognition.

Virtual products refer to the articles that are designed and produced by VR technology, have use value and exchange value, and flow and use in virtual space, with the computer as a creative platform and network as a communication channel. Atoms are the basic units of materials and even physical objects, and the essence of virtual products is a digital model built by a string of binary data in a computer. Virtual products depend on the network virtual space.

We can design packages that can effectively convey the characteristics of virtual products and serve the marketing of virtual products, based on the specific needs of the network, by using VR technology and cutting-edge design concepts, and organically integrating graphics, words, sounds, and other elements. Digital containers and auxiliary materials are used in the circulation and use of virtual products to make them easier to use and manage, to improve the image, and to increase the value and emotional communication, resulting in increased product sales. Virtual packaging is a type of conceptual packaging that is also known as symbol packaging and consciousness packaging. The need for emotional 


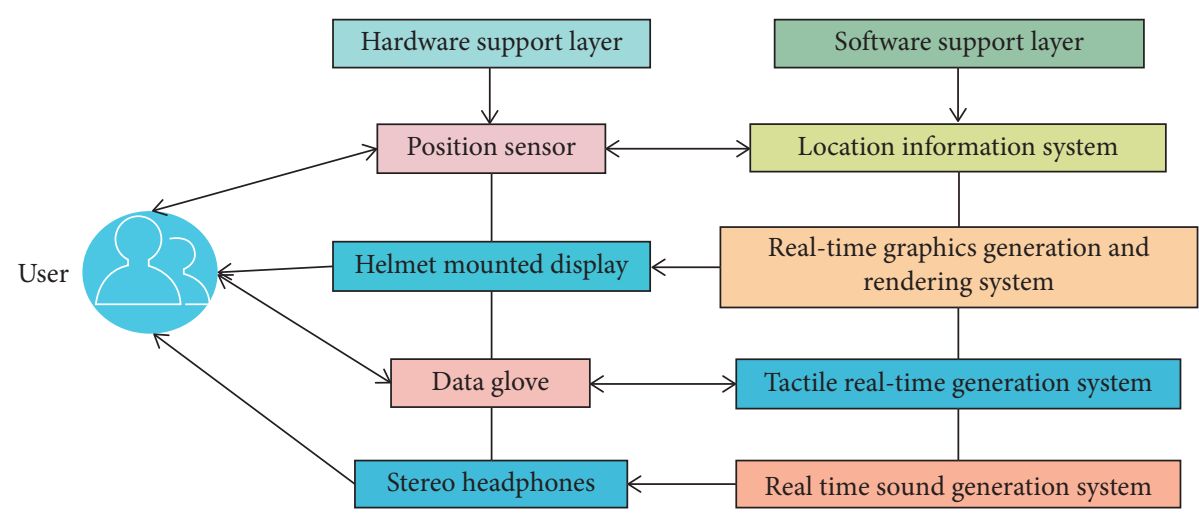

FIGURE 1: Virtual environment architecture.

communication between people and computers, as well as people in the man-machine interface environment, is conveyed by it. Create a VR-based packaging visual graphic element integration module that can scale, roam, and combine visual graphic elements to achieve packaging visual graphic element integration.

\subsection{Graphic Composition Art of Packaging Visual Commu-} nication Design. From the visual communication design itself, graphic engineering is an indispensable part because there is a mutual dependence and mutual achievement between them. In visual communication design, design is often the most important, and composition is the most important supplement. The former is mainly reflected on the surface, while the latter is reflected in the design concept. Graphic art and packaging visual communication design are inextricably linked. Without graphic composition, packaging visual communication design is just empty talk.

The focus of packaging design refers to the concentration of content and the prominence of visual language. Visual expression is to achieve the ideal visual effect through the design of graphics, characters, and colors on the package, so as to realize the promotion function of the package. For product packaging, the design structure should not only show the characteristics of the product concisely but also attract the attention of consumers. It is necessary to be creative and acceptable to people, and the packaging design should be elegant and unconventional. In terms of expression, designers should give full play to the imagination, achieve the highest quality brand image communication effect, and use imagination space, association, abstraction, contrast, and other techniques to complete product packaging design.

Graphic design plays a very important role in commodity packaging. No matter what style of packaging, the key lies in whether there is unique thought and soul, and whether a visual beauty is conveyed through the establishment and application of visual elements such as graphics. In graphic design, "simplicity without monotony, richness without triviality" has always been the highest aesthetic realm pursued by designers. For creative packaging graphics, rich connotation and design artistic conception are particularly important and rare in simple graphics. On the one hand, it is determined by the characteristics of graphics itself to transmit information, and on the other hand, it is to meet the needs of today's fast-paced information society. In the application of graphic composition art in visual communication design, attention should be paid to the application of graphic composition art to shape brand image, convey product information, and attract the audience's attention. Figure 2 shows the cognitive process of packaging design information.

General product packaging design ideas are knowing products, understanding products, feeling products, and creating works. This is the basis of packaging design, and the lack of any link will have an impact on the final design result. The experience of excellent designers comes from accumulated design activities, rich cultural knowledge, and profound composition design literacy, so graphic design, graphic composition, and brand image communication can be closely combined together. As far as the graphic composition is concerned, it is mainly reflected in the plane combination form of visual images; that is to say, it is necessary to decompose and combine regularly according to the formal law of beauty, so as to create a new combination order and form a creative visual image. As far as the composition of graphics is concerned, it highlights the rational characteristics, can reflect a psychological reaction when graphics are transmitted to people through vision, and fully embodies the concept of serving the people and humanized design.

Graphics is a more direct and accurate communication medium in the process of visual communication because it is a symbolic image in the design of visual space. It plays a crucial role in informing and educating people about culture and information. As a result, in packaging visual communication design, the creativity and composition of graphics are rich in artistry. In today's graphic design, people usually pay more attention to how language, words, and sounds are used to create so that graphics can better reflect the characteristics of both sound and emotion, which simply reflects the new concept of combining modern new media technology with broad-based culture. As a result, a mutual dialog mechanism based on communication has been established in a variety of fields, greatly increasing the potential for 


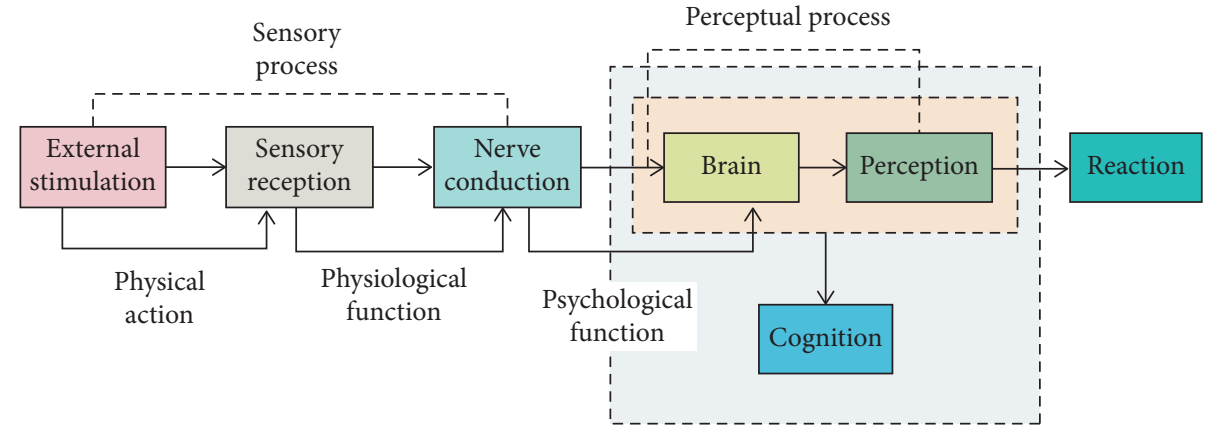

FIGURE 2: Cognitive process of packaging design information.

design reflection, supplement, and description. Visual communication is not a real-time design from this perspective. He emphasizes people's actual information transmission, as well as modern communication ideas and information exchange, with a degree of fact and abstraction that cannot be seen or touched.

\subsection{Packaging Design Based on VR. The form of virtual} packaging is an artificial form designed and processed according to the form of virtual products. It is the skeleton of virtual packaging and the foundation of all other virtual packaging elements. Understanding the virtual packaging form mainly depends on vision and touch, in which the visual response is faster and sharper than the touch response, and it has higher recognition ability for the form. The modeling of virtual packaging does not carry virtual products from the physical level. Although it gets rid of the limitations of space and materials, it should still fit with the theme, form, and emotional level of virtual products. The packaging visual graphic element operation unit can realize the switching, deleting, and adding of packaging visual graphic elements and can help system users obtain the current graphic element existence and its corresponding switching state.

Perform Taylor expansion of the Gaussian difference scale-space function $D(x, y, \sigma)$, where $X=(x, y, \sigma)^{T}$ represents the offset of the original sample point. Then take the derivative of $D(x)$ and set the value to 0 to obtain the extreme value $\hat{x}$ of $x$, as shown in equation (2). Finally, $\hat{x}$ is substituted into equation (1). If the result obtained is less than 0.03 , it is considered as a low-contrast point and deleted.

$$
\begin{aligned}
D(x) & =D+\frac{\partial D^{T}}{\partial x} x+\frac{1}{2} x^{T} \frac{\partial^{2} D}{\partial x^{2}} x, \\
\widehat{x} & =-\frac{\partial^{2} D^{-1}}{\partial x^{2}} \frac{\partial D}{\partial x} .
\end{aligned}
$$

In order to improve the computational efficiency, instead of solving the eigenvalues of the complex matrix, the ratio is calculated. Suppose the larger eigenvalue of the Hessian matrix is $\alpha$, and the smaller eigenvalue is $\beta . r=\alpha / \beta$; the trace of the matrix is $\operatorname{Tr}(H)$. The determinant is $\operatorname{Det}(\mathrm{H})$.

$$
\begin{aligned}
H & =\left[\begin{array}{ll}
D_{x x} & D_{x y} \\
D_{x y} & D_{y y}
\end{array}\right], \\
\operatorname{Tr}(H) & =D_{x x}+D_{y y}=\alpha+\beta, \\
\operatorname{Det}(H) & =D_{x x} D_{y y}-\left(D_{x y}\right)^{2}=\alpha \beta, \\
\text { ratio } & =\frac{\operatorname{Tr}(H)^{2}}{\operatorname{Det}(H)}=\frac{(\alpha+\beta)^{2}}{(\alpha \beta)}=\frac{(\gamma \beta+\beta)^{2}}{\gamma \beta^{2}}=\frac{(\gamma+1)^{2}}{\gamma} .
\end{aligned}
$$

The value of the ratio only depends on $r$ and has nothing to do with the magnitude of $a$ and $\beta$. When $r=1(\alpha=\beta)$, the ratio takes the minimum value; as $r$ increases, the ratio also increases. Therefore, the edge points can be eliminated by calculating the ratio. Lowe regards a ratio greater than or equal to 12.1 as the edge point.

Graphic design, text design, color design, and other elements make up the virtual packaging image. It embodies the communication, promotion, and aesthetics of virtual packaging through the visual and informational design of the exterior of virtual packaging. Virtual packaging image design can reflect the unique characteristics of virtual products, accurately convey information in a short amount of time, attract users' attention, give users an overall impression of products in a relaxed and pleasant environment, and then promote the occurrence of choice and purchase behavior. Importing packaging visual graphic data is possible with the function of adding packaging visual graphic elements. The shapefile that displays packaging visual graphic elements in the file box must be selected in order to perform this function. Read the file in accordance with its specific format, and store it in the appropriate array based on the type of packaging visual graphic elements. Keep track of the number and starting position of the packaging visual graphic elements to make it easier to locate them. Add this file name to the tree control at the end.

Let $S=\{U, C, V, f\}$ be an information system. $X \subseteq C$ is a subset of attributes, where $U / X=\left\{X_{1}, X_{2}, \ldots, X_{n}\right\}$; the information volume of $X$ is defined as

$$
I(X)=\sum_{i=1}^{n} \frac{\left|X_{i}\right|}{|U|}\left[1-\frac{\left|X_{i}\right|}{|U|}\right]=1-\frac{1}{|U|^{2}} \sum_{i=1}^{n}\left|X_{i}\right|^{2}
$$


Among them, $|X|$ represents the cardinality of the set $X$, and $\left|X_{i}\right| /|U|$ represents the probability of the equivalent $X_{i}$ in $\mathrm{U} . \forall x_{i} \in X$, the importance of the conditional attribute $x_{i}$ is defined as

$$
\operatorname{sig}_{X\{x\}}(x)=I(X)-I(X-\{x\}) .
$$

From this, the weight $w_{i}$ of the indicator $x_{i}$ can be expressed as

$$
w_{i}=\frac{\operatorname{sig}_{X\left\{x_{i}\right\}}}{\sum_{i=1}^{n} \operatorname{sig}_{X\left\{x_{i}\right\}}\left(x_{i}\right)}=\frac{I(C)-I\left(C-\left\{c_{i}\right\}\right)}{n I(C)-\sum_{i=1}^{n} I\left(C-\left\{c_{i}\right\}\right)} .
$$

Vision is the most important way for people to obtain external information. The Internet age is an era of eyeball economy, and the sense of vision, which already dominates people's thinking, is more dominant in this era. Virtual packaging, as a new and comprehensive packaging category, conveys to us very rich forms of visual elements, which can be summarized into four main types, namely, images, texts, moving images, and colors. Packaging visual graphic element switch function needs to set the corresponding array of mark element switches and establish the connection between the tree control and the array. When that middle tree item is selected, the corresponding package visual graphic element is displayed; otherwise, the corresponding element cannot be displayed. By controlling the hiding and displaying the state of elements, the display of elements can be turned off and on.

In the packaging visual graphic element style setting unit, the user double-clicks the type identifier in front of each packaging visual graphic element, such as the identifier of a point, and the pattern filling dialog box will pop up in the unit, and then the user can implement classified filling according to the attribute value of the element to change the display style of the element.

\section{Result of Analysis and Discussion}

Build a digital model with tactile information using VR modeling software, and let the experiencer feel the real tactility through virtual devices like data gloves and handles. This is a watershed moment in the evolution of virtual reality technology, enhancing the realism and affinity of the virtual world while embodying the humanized virtual design. How to organize the chosen photos and then realize the composition of the packaging graphics through editing and perfection is a simple task that appears to be difficult to accomplish. Morphological isomorphism, morphological reconstruction, and digital morphology are examples of specific operations. The goal is to combine various form factors. A virtual reality-based visual graphic design system for packaging was created through simulation experiments.

The traditional packaging visual graphic design system is compared to the packaging visual graphic design system based on VR designed in this paper to ensure that the results of this experiment are comparable. Packaging visual graphic design systems based on human-computer interaction, packaging visual graphic design systems based on visual graphics, and packaging visual graphic design systems based on the Internet of Things are examples of traditional packaging visual graphic design systems. Figure 3 shows a comparison of the experimental results of the packaging visual graphic design system's element integration performance based on human-computer interaction, the Internet of Things, and visual graphics.

It can be seen from the figure that the integration of elements based on the visual graphics system has the highest success rate. We conduct comparative experiments on this method and the element integration system based on the VR system. The experimental results of the element integration performance of the packaging visual graphic design system based on VR are shown in Figure 4.

According to the obtained experimental data, the success rate of element integration of packaging visual graphic design systems based on VR is higher than that of packaging visual graphic design systems based on human-computer interaction, visual graphics, and Internet of Things, that is to say, packaging based on VR. The element integration performance of the visual graphic design system is better than that of the traditional packaging visual graphic design system.

In order to further illustrate the effectiveness of the method in this paper, the algorithm in this paper is used to process the samples in the commonly used UCI machine learning database, and the sample Glass data set is selected. The data set contains 300 samples, 10 conditional attributes, and 8 classes. Taking the second attribute in the Glass sample as an example, the data distribution of this attribute is shown in Figure 5.

The element zoom function of the packaging visual graphic design module includes two types: one is to use the mouse to zoom in and zoom out the visual graphic elements of the packaging; the other is to create a rectangular area by dragging the mouse and enlarging the area to enlarge it to the entire screen window to zoom in and out of the element. In the statistics of the experimental results, we eliminated some more concentrated data so that the pixel level distribution of the image is approximately uniform. Perform statistical analysis on the test results. The experimental result data is shown in Figure 6.

The rendering results in the figure show that as the image size grows with the pixel level, the time-consuming of the traditional serial algorithm grows exponentially, while our parallel rendering algorithm grows slowly. It can be seen that our improved parallel rendering algorithm improves the computational efficiency of the original algorithm significantly, and this efficiency improvement will become more apparent as the data scale grows. We will use the rendering of a typical image as an example to look at how rendering efficiency can be improved at each stage of the process. We take one of the packaging design pictures as an example, and the processing time is shown in Figure 7.

The time-consuming gap can be seen to be quite large. In terms of efficiency, the rendering time of each layer of the program that uses parallel rendering is significantly longer than the original algorithm. These results demonstrate that the parallel improved algorithm outperforms the original algorithm in terms of computational efficiency. Virtual reality is essentially a human-computer interaction interface. 


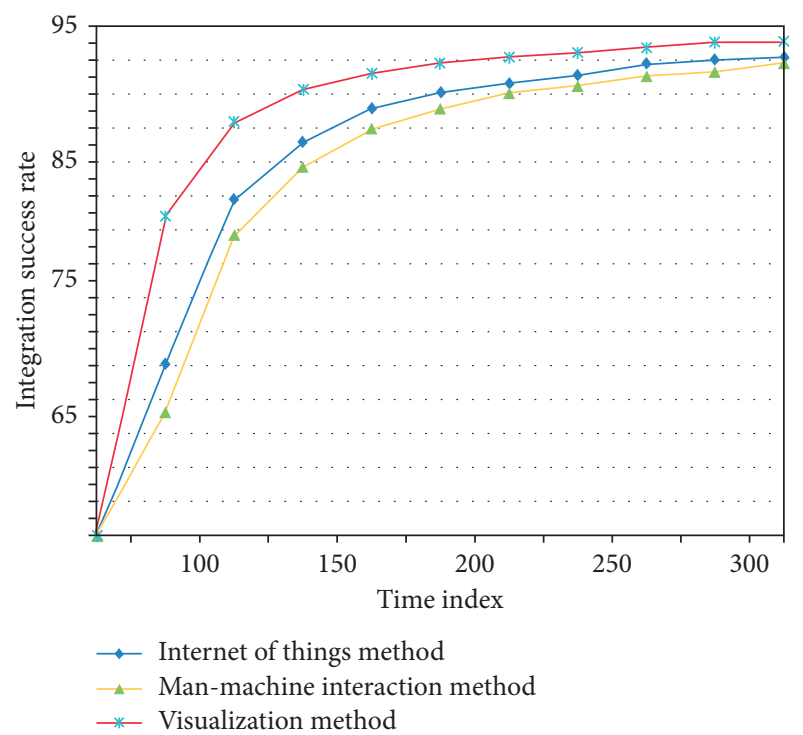

Figure 3: Comparison of experimental results of element integration performance of traditional packaging visual graphic design systems.

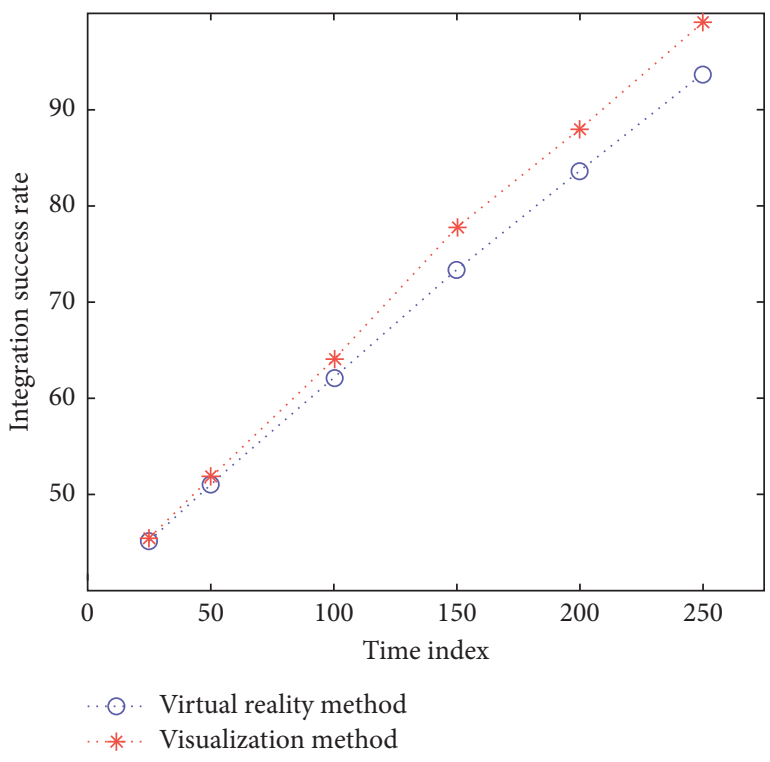

FIgURE 4: Comparison of experimental results of element integration performance between traditional design and VR design.

The requirements for the ease of use of virtual packaging are based on how to integrate the human-machine relationship, enjoy the joy that technology brings us, and feel the true meaning of real life. Users can understand the content to be explained at a glance and choose and edit the information from their own perspective, thanks to the virtual packaging's good interactive performance. On the one hand, the principle of a humanized interactive interface necessitates full consideration of the audience's thinking habits and browsing methods, as well as the proper combination of various elements in terms of information transmission. Users browsing virtual packaging, on the other hand, is a

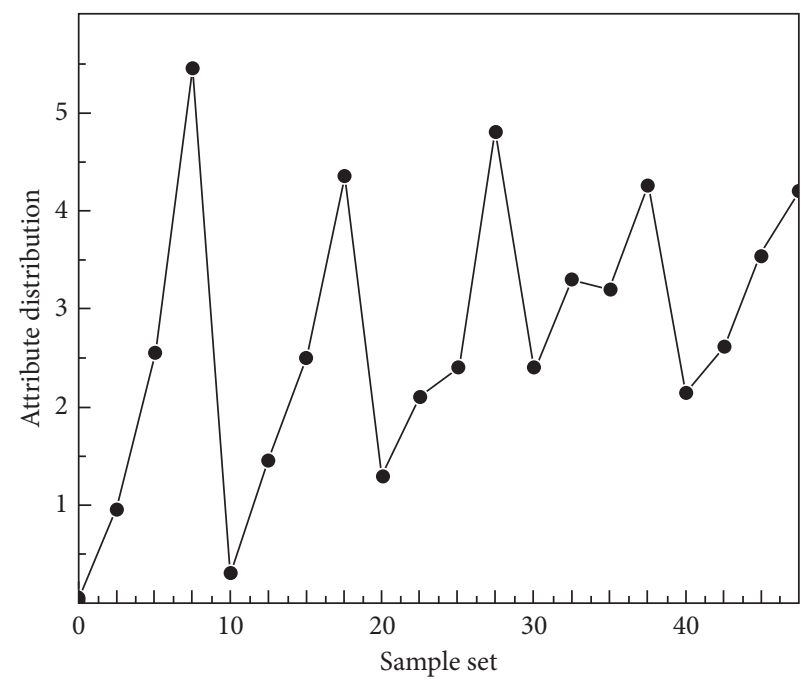

FIGURE 5: Data distribution of the second attribute of the data set.

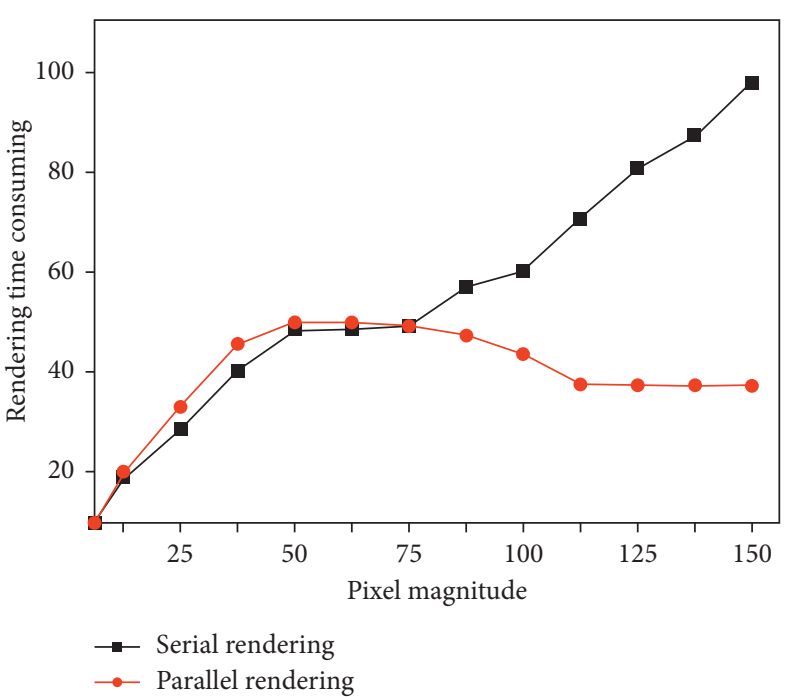

Figure 6: Comparison of experimental data between parallel algorithm and serial algorithm.

nonlinear process that necessitates designers to focus on content layout according to design needs, have a clear hierarchical structure, strengthen the theme's communication, and reduce the difficulty of obtaining information.

After the image processing simulation platform specifies specific rows or columns, it adopts a 3D curved surface projection map to represent the fluctuating state of this information, and the projection map shows a changing curve. Figure 8 is a $3 \mathrm{D}$ surface diagram.

The realization of the element roaming function of the packaging visual graphic design module first needs to calculate the mouse offset, then, the initial position coordinates of the element plus the mouse offset are the origin of the changed element coordinates, and the rectangular range of the element's initial position coordinates reset and redraw the content of the window to realize drag roaming of elements. 


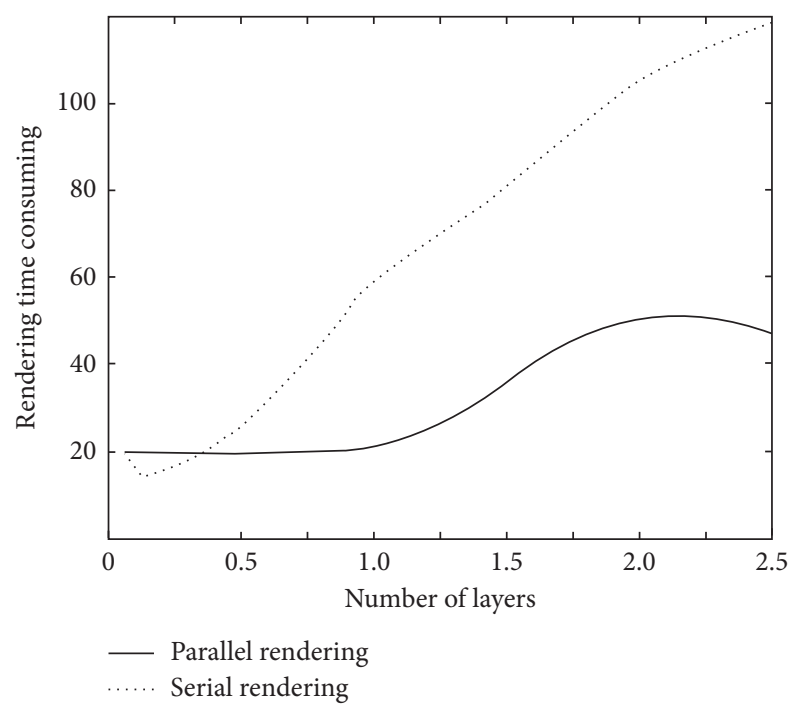

FIGURE 7: Time-consuming comparison of single image rendering.

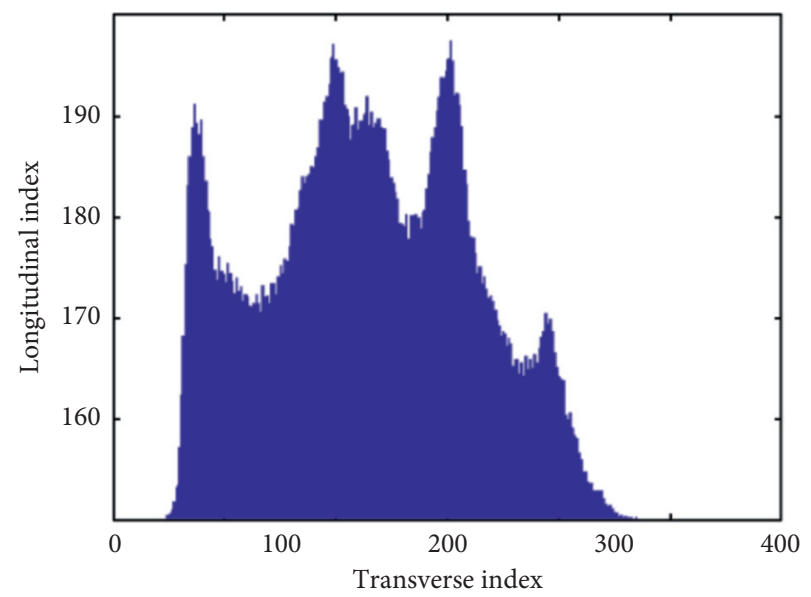

Figure 8: 3D surface projection map output.

This section makes a detailed analysis and summary of related algorithms used in image matching, image stitching, and image fusion. And the simulation experiment of the method is carried out in this paper. The integration of visual graphic elements of packaging is realized through the combination of hardware and software; the element matching module mainly uses the algorithm of this paper to perform element matching. In order to prove that the element integration performance of the system is better, a comparison experiment of element integration performance between the traditional system and the text system is carried out. The experimental results prove that the element integration performance of the system is better than that of the traditional system. The system in this paper realizes the improvement of the element integration design performance, which is of great help to the packaging visual design of various products.

\section{Conclusions}

With the development of society, the concept of design is also developing, and there are more and more interdisciplinary integrations, and the field of visual communication design is also more integrated into other design fields. Visual communication design exists in all aspects of people's lives. Packaging, one of the emerging important marketing elements in this marketing era, plays a unique role in brand promotion. Packaging design is to build a bridge between consumers and products that can communicate with each other. Visual space performance is an important part of modern packaging design. Excellent packaging design is the perfect combination of image and image. It can beautify and weave information and deliver it to people in a pleasing and multisensory way to complete the relationship between products and people. There is an in-depth spiritual dialog. Visual communication design is only a guide, it also requires the perfection and innovation of graphic composition, and the combination of design and composition can produce classic packaging or promotional pictures. The development of science and technology and changes in the economic and cultural environment have brought comprehensive interaction needs to humans. Interaction design has emerged as the times require, and interaction is often realized in a virtual environment. Virtual interaction elements have brought design and various scientific fields. It has a profound influence on thought and method. As an important field in art design, visual communication design should blend with virtual interactive elements, enrich its connotation, and let design blend into life. The application of VR technology in the field of traditional packaging design creatively produces new design concepts and methods that are different from traditional design and achieves design efficiency and design effects that cannot be achieved by traditional packaging design. In terms of the artistic and commercial nature of packaging, both have brought new breakthroughs.

\section{Data Availability}

The data used to support the findings of this study are included within the article.

\section{Conflicts of Interest}

The author does not have any possible conflicts of interest.

\section{References}

[1] S. Serafin, C. Erkut, J. Kojs, N. C. Nilsson, and R. Nordahl, "Virtual reality musical instruments: state of the art, design principles, and future directions," Computer Music Journal, vol. 40, no. 3, pp. 22-40, 2016.

[2] L. Morra, F. Lamberti, F. G. Prattico, S. L. Rosa, and P. Montuschi, "Building trust in autonomous vehicles: role of virtual reality driving simulators in HMI design," IEEE Transactions on Vehicular Technology, vol. 68, no. 10, pp. 9438-9450, 2019.

[3] J.-H. Yin, "A note on packing of graphic n-tuples," Discrete Mathematics, vol. 339, no. 1, pp. 132-137, 2016.

[4] R. Urbas, R. Milošević, N. Kašiković, Ž. Pavlović, and U. S. Elesini, "Microcapsules application in graphic arts industry: a review on the state-of-the-art," Iranian Polymer Journal (English Edition), vol. 26, no. 7, pp. 541-561, 2017. 
[5] F. G. Wu, C. Y. Tseng, and C. M. Cheng, "The composition of visual texture design on surface for color vision deficiency (CVD)," Computers in Human Behavior, vol. 91, no. 2, pp. 84-96, 2018.

[6] J. Lee and J. Song, "The impact of group composition and task design on foreign language learners' interactions in mobilebased intercultural exchanges," ReCALL, vol. 32 , no. 1 , pp. 63-84, 2020.

[7] B. Mehlenbacher, M. K. Autry, and A. R. Kelly, "Instructional design for stem-based collaborative, colocated classroom composition," IEEE Transactions on Professional Communications, vol. 58, no. 4, pp. 396-409, 2016.

[8] A. Horton, "Graphic design visionaries," Technical Communication, vol. 63, no. 4, pp. 380-381, 2016.

[9] A. A. Pochtar and V. V. Malakhov, "Stoichiographic determination of the composition and concentration of phases encapsulated in the bulk of a solid matrix phase," Journal of Analytical Chemistry, vol. 72, no. 7, pp. 719-723, 2017.

[10] A. Prabhu, S. Masghati, P. W. Hernandez, S. J. Kim, and N. C. Klein, "2160 effects of visual fidelity for design of a virtual reality based pain management system," Journal of Minimally Invasive Gynecology, vol. 26, no. 7, p. 165, 2019.

[11] J. Wolfartsberger, "Analyzing the potential of Virtual Reality for engineering design review," Automation in Construction, vol. 104, no. 8, pp. 27-37, 2019.

[12] P. Lo, A. Cho, M.-h. Leung, D. K. W. Chiu, E. H. T. Ko, and K. K. W. Ho, "Use of smartphones by art and design students for accessing library services and learning," Library Hi Tech, vol. 34, no. 2, pp. 224-238, 2016.

[13] G. M. Doniger, M. Plotnik, A. Bahar-Fuchs et al., "Virtual reality-based cognitivemotor training for middle-aged adults at high ad risk: study design and baseline characteristics from a randomized controlled trial," Alzheimer's and Dementia, vol. 13, no. 7, pp. 619-620, 2017.

[14] M. Shannon, "Please make this look nice: the graphic design process," Technical Communication, vol. 64, no. 1, pp. 83-84, 2017.

[15] A. Bateman John, F. O. D. Veloso, J. Wildfeuer, F. H. L. Cheung, and N. S. Guo, "An open multilevel classification scheme for the visual layout of comics and graphic novels: motivation and design," . Digital Scholarship in the Humanities, vol. 32, no. 3, pp. 476-510, 2017.

[16] A. Chung, W. M. To, and B. Schulte-Fortkamp, "Next generation soundscape design using virtual reality technologies," Journal of the Acoustical Society of America, vol. 140, no. 4, p. 3041, 2016.

[17] F. Wei, L. Zheng, H. Deng, and H. Zhang, "Real-time motion tracking for mobile augmented/virtual reality using adaptive visual-inertial fusion," Sensors, vol. 17, no. 5, pp. 1-22, 2017.

[18] L. Brun and A. Gasparini, "Enabling $360^{\circ}$ visual communications: next-level applications and connections," IEEE Consumer Electronics Magazine, vol. 5, no. 2, pp. 38-43, 2016.

[19] R. Dörner, W. Broll, P. Grimm, and B. Jung, "Virtual Reality und Augmented Reality (VR/AR)," Informatik-Spektrum, vol. 39, no. 1, pp. 30-37, 2016.

[20] L. S. Malta, C. Giosan, L. E. Szkodny et al., "Development of a virtual reality laboratory stressor," Virtual Reality, vol. 25, no. 2, pp. 293-302, 2021.

[21] F. Tian, "Immersive $5 \mathrm{G}$ virtual reality visualization display system based on big-data digital city technology," Mathematical Problems in Engineering, vol. 2021, no. 3, pp. 1-9, 2021.

[22] A. Jamali, C. Yousefzadeh, C. Mcginty, D. Bryant, and P. Bos, "LC lens systems to solve accommodation/convergence conflict in three-dimensional and virtual reality displays," Optical Engineering, vol. 57, no. 10, p. 1, 2018.

[23] A. A. Pochtar' and V. V. Malakhov, "New stoichiographic methods for assessing spatial inhomogeneities in the composition and structure of solid substances and materials," Inorganic Materials, vol. 54, no. 7, pp. 749-755, 2018.

[24] M. S. Elbamby, C. Perfecto, M. Bennis, and K. Doppler, "Toward low-latency and ultra-reliable virtual reality," IEEE Network, vol. 32, no. 2, pp. 78-84, 2018.

[25] D. Harvey Christopher, F. Donato, E. I. Moser, and M. Minderer, "FORUM Neuroscience Virtual reality explored," Nature, vol. 533, no. 5, p. 324, 2016.

[26] A. A. Pochtar' and V. V. Malakhov, "Differential dissolution stoichiographic method in investigation of the chemical composition of functional materials," Inorganic Materials, vol. 57 , no. 4 , pp. $435-442,2021$. 